05,11

\title{
Теоретический подход к исследованию магнитных и магнитокалорических свойств сплавов Гейслера $\mathrm{Ni}-\mathrm{Mn}-\mathrm{Ga}$
}

\author{
() О.Н. Мирошкина, В.В. Соколовский, М.А. Загребин, С.В. Таскаев, В.Д. Бучельников \\ Челябинский государственный университет, \\ Челябинск, Россия \\ E-mail: miroshkina.on@yandex.ru
}

Поступила в Редакцию 30 декабря 2019 г.

В окончательной редакции 30 декабря 2019 г.

Принята к публикации 10 января 2020 г.

Представлено исследование магнитных и магнитокалорических свойств сплавов Гейслера $\mathrm{Ni}_{2+x} \mathrm{Mn}_{1-x} \mathrm{Ga}$ $(x=0.16,0.18$ и 0.3$)$. Исследование выполнено с помощью модели, основанной на теории размытых фазовых переходов Малыгина, теории фазовых переходов первого рода Бина-Родбелла и теории молекулярного поля. Исследованы температурные зависимости деформации, намагниченности и изотермического изменения энтропии рассматриваемых сплавов. Показано, что наибольшее изменение магнитной энтропии наблюдается в сплаве $\mathrm{Ni}_{2.18} \mathrm{Mn}_{0.82} \mathrm{Ga}$, в котором мартенситный переход сопровождается сменой магнитного упорядочения. Наименьшее изменение энтропии демонстрирует сплав $\mathrm{Ni}_{2.3} \mathrm{Mn}_{0.7} \mathrm{Ga}$, в котором магнитокалорический эффект наблюдается в мартенситной фазе при смене магнитного упорядочения. Однако, хладоёмкость данного сплава вдвое больше, чем для других рассмотренных композиций.

Ключевые слова: магнитокалорический эффект, сплавы Гейслера, фазовые переходы, многофункциональные материалы.

DOI: $10.21883 /$ FTT.2020.05.49232.22M

\section{1. Введение}

В настоящее время многофункциональные материалы, демонстрирующие магнитокалорический эффект (МКЭ), являются предметом интенсивных фундаментальных и прикладных исследований во всем мире. Интерес к таким материалам обусловлен возможностью их применения в технологии магнитного охлаждения, основанной на МКЭ и являющейся более высокоэффективной и экологически безопасной по сравнению с компрессорными аналогами. Поиск материалов, демонстрирующих высокие значения МКЭ, является одной из ключевых задач материаловедения [1-7]. Редкоземельный металл Gd и $\mathrm{Gd}$-содержащие материалы $\mathrm{Gd}_{5}\left(\mathrm{Si}_{1-x} \mathrm{Ge}_{x}\right)$ [8-10] долгое время рассматривались как наиболее перспективные для использования в магнитном охлаждении. Эти материалы обладают гигантским МКЭ вблизи комнатной температуры, которому отвечает изменение энтропии $\Delta S$ около $12-45 \mathrm{~J} \cdot \mathrm{kg}^{-1} \cdot \mathrm{K}^{-1}$ в сравнительно низких магнитных полях до 2 Т. Кроме того, к материалам с большим МКЭ $\left(\Delta S\right.$ до $30 \mathrm{~J} \cdot \mathrm{kg}^{-1} \cdot \mathrm{K}^{-1}$ при $\left.\Delta \mu_{0} H=1 \mathrm{~T}\right)$ относятся соединения редкоземельных элементов [8-10], соединения на основе $\mathrm{Mn}$ [11-14] и соединения $\mathrm{NaZn}_{13}$-типа [15]. Однако, в последнее время интенсивно исследуются сплавы Гейслера, которые потенциально применимы в технологии магнитного охлаждения в качестве рабочего тела [1-7]. Такие сплавы не содержат токсических элементов, являются более простыми в синтезе и, кроме того, дешевле имеющихся аналогов. Среди сплавов Гейслера можно выделить соединения семейства $\mathrm{Ni}-\mathrm{Mn}-\mathrm{Ga}$, демонстрирующие помимо МКЭ широкое разнообразие функциональных свойств, таких как эффект памяти формы, сверхупругость, сверхпластичность [16-18]. Большинство указанных свойств связано с возможностью структурного перехода между высокотемпературной аустенитной и низкотемпературной мартенситной фазами. Что касается магнитокалорических свойств соединений $\mathrm{Ni}-\mathrm{Mn}-\mathrm{Ga}$, наибольшее значение $\Delta S=20 \mathrm{~J} \cdot \mathrm{kg}^{-1} \cdot \mathrm{K}^{-1}$ при сравнительно небольшом изменении магнитного поля $\Delta \mu_{0} H=1.6 \mathrm{~T}$ наблюдается при связанном магнитоструктурном переходе для соединения $\mathrm{Ni}_{2.19} \mathrm{Mn}_{0.81} \mathrm{Ga}$ [19]. В работе [20] показано, что для $\mathrm{Ni}_{52.6} \mathrm{Mn}_{23.1} \mathrm{Ga}_{24.3}$ изменение энтропии при МКЭ на единицу объема $\Delta S=18 \mathrm{~J} \cdot \mathrm{kg}^{-1} \cdot \mathrm{K}^{-1}$, в то время как для $\mathrm{Gd}_{5} \mathrm{Si}_{2} \mathrm{Ge}_{2}$ и $\mathrm{Gd}$ [9] изменение энтропии при МКЭ составляет $18 \mathrm{~J} \cdot \mathrm{kg}^{-1} \cdot \mathrm{K}^{-1}$ и $9 \mathrm{~J} \cdot \mathrm{kg}^{-1} \cdot \mathrm{K}^{-1}$ соответственно. Авторы работы [21] показали, что в сплавах $\mathrm{Ni}_{2+x} \mathrm{Mn}_{1-x} \mathrm{Ga}(x=0-0.2)$ изменение энтропии $\Delta S$ возрастает практически линейно за счет увеличения магнитного вклада. Указанные особенности МКЭ в сплавах $\mathrm{Ni}-\mathrm{Mn}-\mathrm{Ga}$ обусловлены отклонением от стехиометрического состава и, как следствие, различием температур магнитного и структурного фазовых переходов. Изменяя температуры прямого и обратного структурного фазового перехода при помощи магнитного поля, можно увеличить величину скрытой теплоты перехода на $1-11 \mathrm{~J} / \mathrm{g}$ [22-23]. Структурный фазовый переход в сплавах Ni-Mn-Ga может быть реализован в широком температурном интервале $(4-626 \mathrm{~K})$ и в некоторых случаях может совпадать с магнитным переходом [22,23]. К сожалению, развитие технологии магнитного охлаждения затруднено в связи с температурным гистерезисом, имеющим место в случае фазовых переходов первого рода. Из-за наличия гистерезиса магнитокалорический мате- 
риал теряет свои уникальные свойства после нескольких циклов „нагрев-охлаждение“ и становится неэффективным для использования в качестве хладагента. В связи с этим, большое количество исследований посвящено проблеме поиска способов сужения гистерезиса.

В настоящей работе представлено теоретическое исследование магнитных и магнитокалорических свойств сплавов $\mathrm{Ni}_{2+x} \mathrm{Mn}_{1-x} \mathrm{Ga}(x=0.16,0.18$ и 0.3$)$ с помощью феноменологического подхода, основанного на теории размытых фазовых переходов [24], теории фазовых переходов первого рода Бина-Родбелла [25] и теории молекулярного поля [26]. Выбор композиций обусловлен различными последовательностями фазовых переходов при изменении концентрации избытка $\mathrm{Ni}$.

\section{2. Теоретическая модель}

В настоящей модели описания магнитокалорических свойств сплавов Гейслера во внешнем магнитном поле и поле механических напряжений принимается во внимание сосуществование вблизи точки структурного перехода мартенситного $(m)$ и аустенитного $(a)$ структурных доменов [24]. Введем объемную долю структурной фазы $\alpha$ (где $\alpha=a$ для аустенитных доменов и $\alpha=m$ для мартенситных доменов)

$$
\xi_{\alpha}=V_{\alpha} / V
$$

откуда следует, что

$$
\sum_{\alpha} \xi_{\alpha}=1, \quad 0 \leq \xi_{\alpha} \leq 1 .
$$

Здесь $V_{\alpha}$ - объем фазы $\alpha, V$ - объем всего образца. Объемные доли структурных доменов определяются согласно теории размытых фазовых переходов [24]:

$$
\begin{gathered}
\xi_{m}=\left\{1+\exp \left[\frac { \Delta V Q \rho } { k _ { \mathrm { B } } T _ { m } } \left(\frac{T-T_{m} \pm \Delta T_{m}}{T_{m}}-\frac{\sigma E_{b}}{Q \rho}\right.\right.\right. \\
\left.\left.\left.-\frac{\Delta M \mu_{0} H}{Q}\right)\right]\right\}^{-1}, \quad \xi_{a}=1-\xi_{m},
\end{gathered}
$$

где $\Delta V$ - приращение объема фазы после преодоления межфазной границей одного препятствия, $Q-$ скрытая теплота мартенситного перехода, $\rho$ - плотность сплава, $k_{B}$ - постоянная Больцмана, $T_{m}$ и $\Delta T_{m}-$ температура и полуширина петли гистерезиса мартенситного перехода, $\sigma$ - внешнее механическое напряжение (в рассматриваемой модели - одноосное), $E_{b}-$ деформация Бейна, $\Delta M-$ изменение намагниченности при структурном переходе и $\mu_{0} H-$ внешнее магнитное поле. Поскольку структурное превращение в рассматриваемых сплавах является фазовым переходом первого рода, оно сопровождается гистерезисом вследствие взаимодействия межфазных поверхностей с препятствиями в решетке. Это обстоятельство учитывается знаком слагаемого $\pm \Delta T_{m}$, где знак „ $+{ }^{“}$ относится к прямому мартенситному переходу, а „-““ к обратному. Согласно теории размытых фазовых переходов Малыгина [24], ширина гистерезиса зависит от концентрации дефектов $C_{\mathrm{def}}$, препятствующих движению межфазных границ в кристалле, и связана с ней следующим образом

$$
\Delta T_{m}=\frac{3 k_{\mathrm{B}} T_{m}^{2}}{\pi \rho Q r^{3}} C_{\mathrm{def}},
$$

где $r$ - радиус дефекта. Взаимодействие межфазных границ с дефектами является причиной размытия мартенситного перехода и возникновения гистерезиса.

Плотность свободной энергии кристалла учитывает аустенитный и мартенситный вклады

$$
F=\xi_{m} F_{m}+\xi_{a} F_{a}-\sigma E .
$$

Плотность свободной энергии фазы $\alpha$ содержит слагаемые, ответственные за упругую (el), магнитную (mag) и магнитоупругую (me) части:

$$
F_{\alpha}=F_{\alpha}^{\mathrm{el}}+F_{\alpha}^{\mathrm{mag}}+F_{\alpha}^{\mathrm{me}} .
$$

Упругая часть свободной энергии получена путем интегрирования выражений, связывающих плотность свободной энергии с модулем упругости $C$, коэффициентом теплового расширения $\varsigma$ и удельной теплоемкости $c$ [27]. В предложенной модели полагается, что $C, \varsigma$ и $c$ не зависят от температуры и для аустенитной и мартенситной фазы равны друг другу. Дважды продифференцировав выражение для свободной энергии, получим, что модулю упругости $C$ в рассматриваемой модели соответствует модуль всестороннего сжатия. Известно, что модуль всестороннего сжатия уменьшается практически вдвое вблизи точки плавления, в то время как он остается практически неизменным при температурах, близких к температурам структурного перехода. Таким образом, правомерно при расчетах полагать $C$ постоянным. Магнитная часть свободной энергии $F_{\alpha}^{\mathrm{mag}}$ определяется в рамках теории молекулярного поля, а магнитоупругая часть $F_{\alpha}^{\text {me }}$ - в рамках теории Бина-Родбелла [25].

Таким образом, слагаемые, выходящие в выражение для свободной энергии, имеют следующий вид

$$
\begin{gathered}
F_{a}^{\mathrm{el}}=\frac{C E^{2}}{2}-\varsigma C E\left(T-T_{m}\right)+\rho c T\left(1-\ln \frac{T}{T_{m}}\right), \\
F_{m}^{\mathrm{el}}=\frac{C\left(E+E_{b}\right)^{2}}{2}-\varsigma C\left(E+E_{b}\right)\left(T-T_{m}\right) \\
\quad+\rho c T\left(1-\ln \frac{T}{T_{m}}\right)-Q \rho\left(1-\frac{T}{T_{m}}\right), \\
F_{\alpha}^{\mathrm{mag}}=-\left(\frac{A_{\alpha} y_{\alpha}^{2}}{2}+\mu_{0} H M_{0 \alpha} y_{\alpha}\right) \frac{R \rho}{\mu}-\rho T S_{\alpha}^{\mathrm{mag}}, \\
F_{a}^{\mathrm{me}}=\frac{B_{a} y_{a}^{2} E}{2} \frac{R \rho}{\mu}, \quad F_{m}^{\mathrm{me}}=\frac{B_{m} y_{m}^{2}\left(E+E_{b}\right)}{2} \frac{R \rho}{\mu} .
\end{gathered}
$$

Здесь $A_{\alpha}$ - параметр обменного взаимодействия, $M_{0 \alpha}-$ намагниченность насыщения, $y_{\alpha}-$ относительная намагниченность, $R-$ универсальная газовая постоянная, 
$\mu$ - молярная масса соединения, $S_{\alpha}^{\mathrm{mag}}-$ магнитная энтропия, $B_{\alpha}-$ константа магнитострикции, $E-$ деформация.

Параметр обменного взаимодействия и намагниченность насыщения определяются из выражений

$$
A_{\alpha}=\frac{3 J_{\alpha} T_{C \alpha}}{J_{\alpha}+1}, \quad M_{0 \alpha}=\frac{\mu_{B} g_{\alpha} J_{\alpha}}{k_{\mathrm{B}}}
$$

где $J_{\alpha}-$ полный угловой момент, $T_{C \alpha}-$ температура Кюри, $g_{\alpha}-$ фактор Ланде и $\mu_{\mathrm{B}}-$ магнетон Бора.

Константа магнитострикции $B_{\alpha}$ в приближении молекулярного поля задается следующим выражением:

$$
B_{\alpha}=\sqrt{\frac{18 C\left(\left(2 J_{\alpha}+1\right)^{4}-1\right)}{80\left(J_{\alpha}+1\right)^{4}} T_{C \alpha} \eta_{\alpha} \frac{\mu}{R \rho}},
$$

где параметр $\eta_{\alpha}$ определяет род фазового перехода $[25,26]$. Из теории фазовых переходов Бина-Родбелла следует условие, что при $\eta_{\alpha}>1$ в магнитной системе осуществляется фазовый переход I рода, в то время как при $\eta_{\alpha}<1$ имеет место фазовый переход II рода.

Для определения деформации $E$ и магнитного параметра порядка $y$ необходимо проминимизировать выражение для свободной энергии (2) сначала по $E$, а затем по $y$. В результате, уравнение для относительной намагниченности может быть записано с использованием функции Бриллюэна $B_{J}$

$$
\begin{aligned}
y_{\alpha} & =B_{J} Y_{\alpha} \\
& =\frac{2 J_{\alpha}+1}{2 J_{\alpha}} \operatorname{coth}\left[\frac{\left(2 J_{\alpha}+1\right) Y_{\alpha}}{2 J_{\alpha}}\right]-\frac{1}{2 J_{\alpha}} \operatorname{coth}\left(\frac{Y_{\alpha}}{2 J_{\alpha}}\right),
\end{aligned}
$$

где

$$
\begin{gathered}
Y_{\alpha}=\frac{1}{T}\left[\left(A_{\alpha}+\varsigma B_{\alpha}\left(T-T_{m}\right)+\frac{\sigma B_{\alpha}}{C}\right) y_{\alpha}\right. \\
\left.+\frac{R \rho}{\mu} \frac{B_{\alpha}^{2} y_{\alpha}^{3}}{2 C}+M_{0 \alpha} \mu_{0} H\right] .
\end{gathered}
$$

Таким образом, намагниченность всего образца

$$
\begin{gathered}
M=\frac{R}{\mu}\left(M_{0 a} \xi_{a} y_{a} \frac{H}{H_{a}^{\text {coer }}}+M_{0 m} \xi_{m} y_{m} \frac{H}{H_{m}^{\text {coer }}}\right) \\
\text { при } H<H_{\mathrm{all}}^{\text {coer }} \\
M=\frac{R}{\mu}\left(M_{0 a} \xi_{a} y_{a}+M_{0 m} \xi_{m} y_{m}\right) \text { при } H>H_{\mathrm{all}}^{\text {coer }},
\end{gathered}
$$

где $H_{\mathrm{all}}^{\mathrm{coer}}=H_{a}^{\mathrm{coer}}+H_{m}^{\mathrm{coer}}-$ коэрцитивная сила соединения.

Вычислив относительную намагниченность $y_{\alpha}$, можно рассчитать деформацию образца

$$
E=\frac{R \rho}{\mu} \frac{\left(B_{a} \xi_{a} y_{a}^{2}+B_{m} \xi_{m} y_{m}^{2}\right)}{2 C}+\varsigma\left(T-T_{m}\right)+\xi_{m} E_{b}+\frac{\sigma}{C} .
$$

Магнитная часть энтропии может быть найдена в рамках теории молекулярного поля

$$
S^{\mathrm{mag}}=S_{a}^{\mathrm{mag}} \xi_{a}+S_{m}^{\mathrm{mag}} \xi_{m},
$$

где

$$
S_{\alpha}^{\operatorname{mag}}=\frac{R}{\mu}\left[\ln \frac{\sinh \left(\frac{2 J_{\alpha}+1}{2 J_{\alpha}} Y_{\alpha}\right)}{\sinh \left(\frac{Y_{\alpha}}{2 J_{\alpha}}\right)}-y_{\alpha} Y_{\alpha}\right] .
$$

Для расчета изотермической энтропии с переменным внешним магнитным полем используется нижеприведенное выражение

$$
\Delta S^{\mathrm{mag}}\left(T, \mu_{0} H\right)=S^{\mathrm{mag}}\left(T, \mu_{0} H\right)-S^{\mathrm{mag}}(T, 0) .
$$

Здесь $S^{\mathrm{mag}}\left(T, \mu_{0} H\right)$ и $S^{\mathrm{mag}}(T, 0)$ - энтропия в магнит-

\begin{tabular}{|c|c|c|c|}
\hline Композиция & $x=0.16$ & $x=0.18$ & $x=0.3$ \\
\hline \multicolumn{4}{|c|}{ Структурные свойства } \\
\hline$a_{0}[\AA]$ & 5.830 & 5.825 & 5.815 \\
\hline$\mu[\mathrm{kg} / \mathrm{mol}]$ & 0.2426 & 0.2427 & 0.2432 \\
\hline$\rho\left[\mathrm{kg} / \mathrm{m}^{3}\right]$ & 8178 & 8181 & 8196 \\
\hline$T_{m}[\mathrm{~K}]$ & 314 & 335 & 530 \\
\hline$\Delta T_{m}[\mathrm{~K}]$ & 3.5 & 3.0 & - \\
\hline$\Delta V\left[\mathrm{~m}^{3}\right]$ & \multicolumn{3}{|c|}{$5 \cdot 10^{-26}$} \\
\hline$Q[\mathrm{~J} / \mathrm{kg}]$ & \multicolumn{3}{|c|}{7000} \\
\hline$C[\mathrm{GPa}]$ & \multicolumn{3}{|c|}{150} \\
\hline \multicolumn{4}{|c|}{ Магнитные свойства } \\
\hline$T_{C a}[\mathrm{~K}]$ & 338 & 320 & 0 \\
\hline$T_{C m}[\mathrm{~K}]$ & 368 & 355 & 350 \\
\hline$\Delta M\left[\mathrm{~A} \cdot \mathrm{m}^{2} / \mathrm{kg}\right]$ & 22 & 40 & 0 \\
\hline$g_{a}$ & 2.4 & 2.5 & 2.4 \\
\hline$g_{m}$ & 2.0 & 2.0 & 2.0 \\
\hline$J_{a}$ & 1.9 & 1.4 & 1.56 \\
\hline$J_{m}$ & 1.9 & 1.4 & 1.59 \\
\hline
\end{tabular}
ном поле $\mu_{0} H$ и в нулевом поле.

\section{3. Детали расчетов}

С помощью представленной модели проведено исследование магнитных и магнитокалорических свойств для трех композиций семейства $\mathrm{Ni}-\mathrm{Mn}-\mathrm{Ga}$, принадлежащих трем разным областям $(T-x)$ фазовой диаграммы и иллюстрирующих различные последовательности фазовых переходов. В качестве первого образца выбрано соединение $\mathrm{Ni}_{2.16} \mathrm{Mn}_{0.84} \mathrm{Ga}$, демонстрирующее разделенные магнитный и структурный переходы. В данном

Таблица 1. Параметры модели для сплавов $\mathrm{Ni}_{2+x} \mathrm{Mn}_{1-x} \mathrm{Ga}$ $(x=0.16,0.18$ и 0.3$)$

Параметр модели Бина-Родбелла

\begin{tabular}{c|l|l|l}
\hline$\eta_{a}$ & 0.1 & 1.4 & 1.0 \\
\hline$\eta_{m}$ & 1.3 & 1.4 & 0.1
\end{tabular}



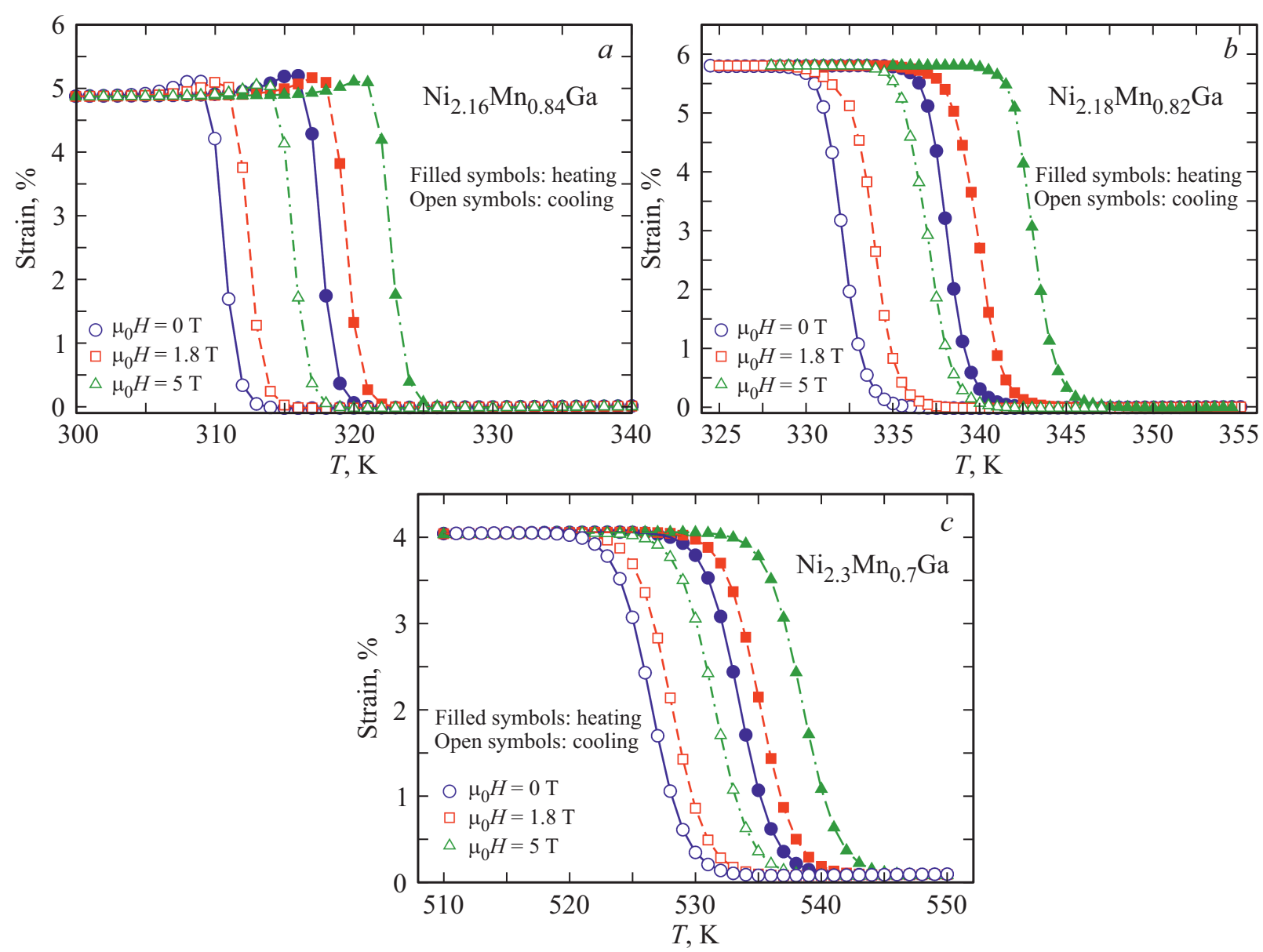

Рис. 1. Температурные зависимости деформации во внешних магнитных полях $0,1.8$ и $5 \mathrm{~T}$ для сплавов $(a) \mathrm{Ni}_{2.16} \mathrm{Mn}_{0.84} \mathrm{Ga}_{\text {, }}$ (b) $\mathrm{Ni}_{2.18} \mathrm{Mn}_{0.82} \mathrm{Ga}$ и $(c) \mathrm{Ni}_{2.3} \mathrm{Mn}_{0.7} \mathrm{Ga}$ при охлаждении и нагреве.

случае при нагреве реализуется сначала мартенситный переход I рода в ферромагнитном (ФМ) состоянии, а затем - магнитный фазовый переход II рода из ФМ в парамагнитное $(П \mathrm{M})$ состояние в аустенитной фазе. В качестве второго образца рассмотрен $\mathrm{Ni}_{2.18} \mathrm{Mn}_{0.82} \mathrm{Ga}$, в котором имеет место связанный магнитоструктурный переход I рода, т. е. мартенситный переход сопровождается сменой магнитного упорядочения из ФМ в ПМ состояние. В качестве третьего образца взят $\mathrm{Ni}_{2.3} \mathrm{Mn}_{0.7} \mathrm{Ga}$, в котором при нагреве сначала происходит магнитный фазовый переход II рода из ФМ в ПМ состояние в мартенситной фазе и последующий структурный переход из ПМ мартенсита в ПМ аустенит.

Входные параметры модели для рассматриваемых соединений $\mathrm{Ni}_{2+x} \mathrm{Mn}_{1-x} \mathrm{Ga}(x=0.16,0.18$ и 0.3$)$ представлены в табл. 1. Плотность соединений $\rho$ вычислена как отношение молярной массы вещества к молярному объему. Оценка величины скрытой теплоты перехода произведена на основе результатов эксперимента [28]. Температуры магнитных и структурных переходов, а также величина полуширины гистерезиса взяты из экспериментов [29] и [30]. Величина модулей упругости оценена из первопринципного исследования [31]. Мо- делирование проводилось для случаев $H>H_{\text {all }}^{\text {coer }}$ (уравнение (3)).

\section{4. Результаты и обсуждение}

В настоящем разделе представлены результаты исследования магнитных и магнитокалорических свойств сплавов $\mathrm{Ni}_{2+x} \mathrm{Mn}_{1-x} \mathrm{Ga}$ с помощью предложенной модели. На рис. 1 приведены зависимости деформации кристаллической решетки от температуры вблизи мартенситного перехода. Видно, что приложение внешнего магнитного поля ведет к смещению величины температуры структурного перехода в область более высоких значений с шагом $d T_{m} / d H \approx 1 \mathrm{~K} / \mathrm{T}$ в соответствии c экспериментом. Наибольшую величину деформации демонстрирует сплав $\mathrm{Ni}_{2.18} \mathrm{Mn}_{0.82} \mathrm{Ga}$, в то время как увеличение избытка $\mathrm{Ni}$ до $x=0.3$ ведет к уменьшению деформации, что, вероятно, обусловлено высокими температурами мартенситного перехода.

Температурные зависимости намагниченности сплавов $\mathrm{Ni}_{2+x} \mathrm{Mn}_{1-x} \mathrm{Ga}$ в различных магнитных полях представлены на рис. 2. Из данных зависимостей видно, что 

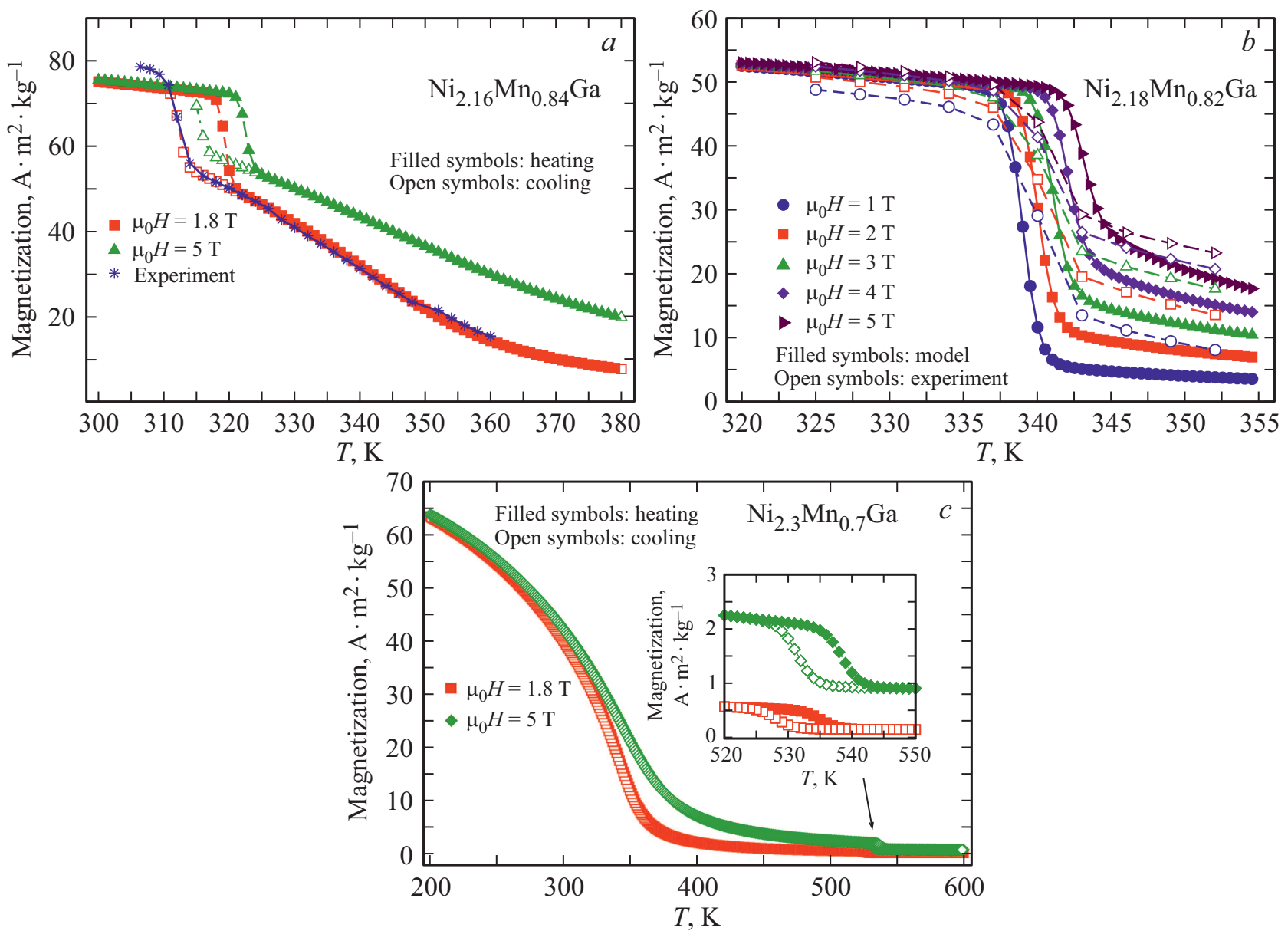

Рис. 2. Температурные зависимости намагниченности для сплавов (a) $\mathrm{Ni}_{2.16} \mathrm{Mn}_{0.84} \mathrm{Ga}$, (b) $\mathrm{Ni}_{2.18} \mathrm{Mn}_{0.82} \mathrm{Ga}$ и $(c) \mathrm{Ni}_{2.3} \mathrm{Mn}_{0.7} \mathrm{Ga}_{\text {. }}$ Экспериментальные данные для случаев $x=0.16$ и $x=0.18$ взяты из работ [30] и [33]. Для сплава $\mathrm{Ni}_{2.18} \mathrm{Mn}_{0.82} \mathrm{Ga}$ теоретические и экспериментальные кривые приведены при нагреве.

увеличение внешнего магнитного поля приводит к смещению температуры структурного перехода и перегиба намагниченности в область более высоких температур. Для случая $\mathrm{Ni}_{2.16} \mathrm{Mn}_{0.84} \mathrm{Ga}$ (рис. 2,a) в магнитном поле $1.8 \mathrm{~T}$ мартенситный переход происходит в ФМ фазе при температуре $316 \mathrm{~K}$ и сопровождается температурным гистерезисом. Усиление магнитного поля до $5 \mathrm{~T}$ приводит к увеличению температуры структурного перехода до $319 \mathrm{~K}$ и уменьшению скачка намагниченности на $4.6 \mathrm{~A} \cdot \mathrm{m}^{2} / \mathrm{kg}$ по сравнению со случаем поля $1.8 \mathrm{~T}$. Кроме того, усиление магнитного поля повышает намагниченность аустенита. Следует отметить, что такое поведение намагниченности наблюдается при условии $T_{m}<T_{C a}<T_{C m}$ согласно работе [32]. Результаты моделирования хорошо согласуются с имеющимися экспериментальными данными для случая охлаждения образца в поле $1.8 \mathrm{~T}$.

Вторая композиция $\mathrm{Ni}_{2.18} \mathrm{Mn}_{0.82} \mathrm{Ga}$ принадлежит области фазовой диаграммы $\mathrm{Ni}-\mathrm{Mn}-\mathrm{Ga}$, характеризующейся температурами переходов $T_{C} \approx T_{m}$. На рис. $2, b$ представлены теоретические и экспериментальные кривые намагниченности данного сплава при нагреве в полях от 1 до 5T. Экспериментальные данные были получены из набора кривых $M\left(\mu_{0} H\right)$ [33]. Сопоставляя данный рисунок с рис. $1, b$, можно видеть, что в этом случае реализуется связанный магнитоструктурный фазовый переход, температура которого увеличивается с $339 \mathrm{~K}$ в поле $1 \mathrm{~T}$ до $343 \mathrm{~K}$ в $5 \mathrm{~T}$. Кроме того, как и в случае $x=0.16$, увеличение магнитного поля уменьшает скачок намагниченности в области фазового перехода.

Результаты моделирования температурных зависимостей намагниченности для третьей композиции представлены на рис. 2, c. Видно, что в сплаве $\mathrm{Ni}_{2.3} \mathrm{Mn}_{0.7} \mathrm{Ga}$ происходит магнитный переход II рода, характеризующийся сменой ФМ упорядочения на ПМ и отсутствием гистерезиса. Однако, при структурном переходе, имеющем место в ПМ фазе,наблюдается несущественное изменение намагниченности, сопровождающееся гистерезисом.

Исследование магнитокалорических свойств сплавов $\mathrm{Ni}_{2+x} \mathrm{Mn}_{1-x} \mathrm{Ga}$ проведено посредством вычисления изменения магнитной части энтропии при изменении магнитного поля от 0 до 1.8 и 5 Т для случаев нагрева и охлаждения. Кроме того, проведено сравнение результа- 

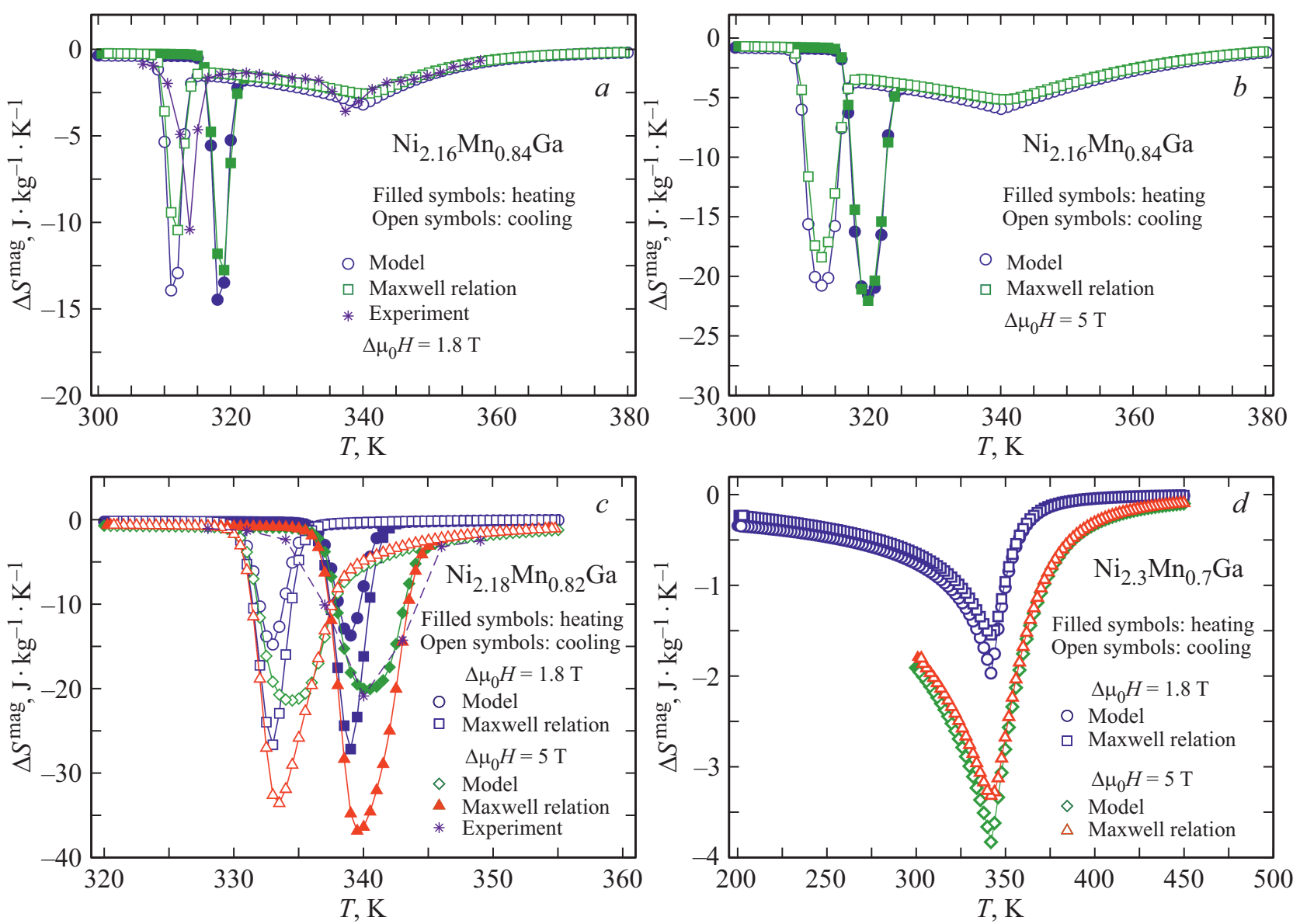

Рис. 3. Температурные зависимости изменения магнитной энтропии во внешних магнитных полях 1.8 и 5 Т для сплавов $(a, b) \mathrm{Ni}_{2.16} \mathrm{Mn}_{0.84} \mathrm{Ga},(c) \mathrm{Ni}_{2.18} \mathrm{Mn}_{0.82} \mathrm{Ga}$ и $(d) \mathrm{Ni}_{2.3} \mathrm{Mn}_{0.7} \mathrm{Ga}$. Экспериментальные данные для случаев $x=0.16$ и $x=0.18$ взяты из работ [30] и [33].

тов моделирования с расчетами МКЭ из соотношения Максвелла

$$
\Delta S^{\mathrm{mag}}\left(T, \mu_{0} H\right)=\mu_{0} \int_{0}^{H}\left(\frac{\partial M}{\partial T}\right) d H .
$$

В данном случае набор кривых $M\left(\mu_{0} H\right)$ был получен с шагом $0.1 \mathrm{~T}$.

Из рис. 3 видно, что усиление магнитного поля с 1.8 до $5 \mathrm{~T}$ ведет к увеличению МКЭ и уширению пиков $\Delta S$, что свидетельствует о увеличении хладоемкости рассматриваемых сплавов. Для композиций $x=0.16$ и 0.18 МКЭ становится более ярко выраженным, возрастая примерно на $7 \mathrm{~J} \cdot \mathrm{kg}^{-1} \cdot \mathrm{K}^{-1}$. Изменение энтропии в поле 5 Т для этих сплавов равно -20.7 и $-21.4 \mathrm{~J} \cdot \mathrm{kg}^{-1} \cdot \mathrm{K}^{-1}$, соответственно, что делает их интересными для рассмотрения в качестве хладагентов. В отличие от них, для сплава $\mathrm{Ni}_{2.3} \mathrm{Mn}_{0.7} \mathrm{Ga}$ изменение энтропии возрастает на $-1.8 \mathrm{~J} \cdot \mathrm{kg}^{-1} \cdot \mathrm{K}^{-1}$ при увеличении поля от 1.8 до $5 \mathrm{~T}$. Что касается сравнения с расчетами из соотношения Максвелла, из рис. $3, a, b$ видно, что результаты моделирования для $\mathrm{Ni}_{2.16} \mathrm{Mn}_{0.84} \mathrm{Ga}$ дают слегка завышенные значения МКЭ по сравнению с соотношением
Максвелла и экспериментом [30]. Обратная ситуация наблюдается для сплава со связанным магнитоструктурным переходом $\mathrm{Ni}_{2.18} \mathrm{Mn}_{0.82} \mathrm{Ga}$ (рис. 3,c). В этом случае результаты моделирования хорошо согласуются с экспериментом [30], тогда как соотношение Максвелла дает завышенное значение МКЭ, что обусловлено резким изменением теоретически рассчитанных кривых намагниченности в точке структурного перехода по сравнению с более пологими экспериментальными кривыми. В целом, величина МКЭ в соединениях $\mathrm{Ni}_{2.16} \mathrm{Mn}_{0.84} \mathrm{Ga}$ и $\mathrm{Ni}_{2.18} \mathrm{Mn}_{0.82} \mathrm{Ga}$ при охлаждении немного меньше, чем при нагреве.

Однако, стоит отметить, что большое изменение магнитной энтропии не являтся достаточным показателем эффективности хладагента. Важной характеристикой является также ширина температурного диапазона, в котором наблюдается МКЭ. Для оценки материала по этому критерию используется хладоемкость (refrigeration capacity, RC), показывающая, какое количество теплоты может быть отведено при охлаждении $1 \mathrm{~kg}$ материала за один цикл при определенном изменении магнитного поля. Результаты расчетов хладоемкости сплавов $\mathrm{Ni}_{2+x} \mathrm{Mn}_{1-x} \mathrm{Ga}(x=0.16,0.18$ и 0.3$)$ представлены в 
Таблица 2. Магнитокалорические свойства сплавов $\mathrm{Ni}_{2+x} \mathrm{Mn}_{1-x} \mathrm{Ga} \quad(x=0.16, \quad 0.18$ и 0.3$), \quad$ полученные $\mathrm{c}$ помощью представленной модели (Модель), соотношения Максвелла (Максвелл) и из эксперимента (Эксп.). (Здесь $\Delta S\left(\mathrm{~J} \cdot \mathrm{kg}^{-1} \cdot \mathrm{K}^{-1}\right)$ - изменение магнитной части энтропии при фазовом переходе, $\delta T(\mathrm{~K})-$ разность температур на половине высоты пика $\Delta S, R C=\Delta S \delta T\left(\mathrm{~J}^{\circ} \mathrm{kg}^{-1}\right) \quad$ хладоемкость соединения

\begin{tabular}{c|l|c|c|c|c|c|c}
\hline \multirow{2}{*}{} & \multicolumn{3}{|c|}{$\Delta \mu_{0} H=1.8 \mathrm{~T}$} & \multicolumn{3}{c}{$\Delta \mu_{0} H=5 \mathrm{~T}$} \\
\cline { 3 - 8 } \multicolumn{2}{c|}{} & $\Delta S$ & $\delta T$ & $R C$ & $\Delta S$ & $\delta T$ & $R C$ \\
\hline \multirow{3}{*}{$\mathrm{Ni}_{2.16} \mathrm{Mn}_{0.84} \mathrm{Ga}$} & Модель & -14.0 & 2.6 & 36.4 & -20.7 & 5.4 & 111.8 \\
& Максвелл & -10.5 & 2.6 & 27.3 & -18.4 & 4.9 & 90.2 \\
& Эксп. & -10.4 & 2.7 & 28.1 & - & - & - \\
\hline \multirow{3}{*}{$\mathrm{Ni}_{2.18} \mathrm{Mn}_{0.82} \mathrm{Ga}$} & Модель & -15.0 & 2.6 & 39.0 & -20.0 & 5.2 & 104.0 \\
& Максвелл & -26.5 & 2.4 & 63.6 & -36.7 & 4.7 & 172.5 \\
& Эксп. & - & - & - & -21.0 & 7.0 & 147.0 \\
\hline \multirow{2}{*}{$\mathrm{Ni}_{2.3} \mathrm{Mn}_{0.7} \mathrm{Ga}$} & Модель & -2.0 & 40.3 & 79.8 & -3.8 & 57.6 & 218.9 \\
& Максвелл & -1.6 & 41.2 & 65.9 & -3.3 & 62.8 & 207.2
\end{tabular}

табл. 2. Несмотря на то, что сплав $\mathrm{Ni}_{2.3} \mathrm{Mn}_{0.7} \mathrm{Ga}$ обладает наименьшим изменением намагниченности, хладоемкость данного сплава вдвое больше по сравнению с $\mathrm{Ni}_{2.16} \mathrm{Mn}_{0.84} \mathrm{Ga}$ и $\mathrm{Ni}_{2.18} \mathrm{Mn}_{0.82} \mathrm{Ga}$. Это связано с тем, что интервал температур, в котором происходит МКЭ, на порядок больше по сравнению с другими рассматриваемыми сплавами серии.

\section{5. Заключение}

В настоящей работе представлено теоретическое исследование магнитных и магнитокалорических свойств сплавов Гейслера $\mathrm{Ni}_{2+x} \mathrm{Mn}_{1-x} \mathrm{Ga}(x=0.16,0.18$ и 0.3$)$, выполненное с помощью модели, основанной на теории размытых фазовых переходов Малыгина, теории фазовых переходов первого рода Бина-Родбелла и теории молекулярного поля. В результате исследования температурных зависимостей деформации и намагниченности установлено, что магнитное поле приводит к смещению температуры мартенситного перехода и перегиба намагниченности в область более высоких температур. Расчеты магнитокалорических свойств $\mathrm{Ni}_{2+x} \mathrm{Mn}_{1-x} \mathrm{Ga}(x=0.16,0.18$ и 0.3$)$ показывают, что наибольшее изменение магнитной части энтропии наблюдается для композиций $x=0.16$ и 0.18 . Однако, наибольшей хладоемкостью обладает соединение $x=0.3$, которое, несмотря на небольшое изменение энтропии при магнитном переходе, демонстрирует МКЭ в более широком температурном диапазоне вследствие фазового перехода II рода. Проведенное исследование показало, что сплавы $\mathrm{Ni}_{2+x} \mathrm{Mn}_{1-x} \mathrm{Ga}(x=0.16,0.18$ и 0.3$)$ могут считаться перспективными кандидатами для использования в качестве хладагентов в технологии магнитного охлаждения.

\section{Финансирование работы}

Работа выполнена при поддержке гранта Российского фонда фундаментальных исследований № 18-3200507 мол_а.

\section{Конфоликт интересов}

У авторов статьи нет конфликта интересов.

\section{Список литературы}

[1] K.A. Gschneidner, Jr., V.V. Pecharsky, A.O. Tsokol. Rep. Prog. Phys. 68, 1479 (2005).

[2] K.A. Gschneidner, Jr., V.V. Pecharsky. Int. J. Refrig. 31, 945 (2008).

[3] A. Planes, L1. Mañosa, M. Acet. J. Phys.: Condens. Matter 21, 233201 (2009).

[4] V. Franco, J.S. Blázquez, B. Ingalge, A. Conde. Annu. Rev. Mater. Res. 42, 305 (2012).

[5] N.A. de Oliveira, P.J. von Ranke, A. Troper. Int. J. Refrig. 37, 237 (2014).

[6] X. Moya, S. Kar-Narayan, N.D. Mathur. Nature Mater. 13, 439 (2014).

[7] V.V. Khovaylo, V.V. Rodionova, S.N. Shevyrtalov, V. Novosad. Phys. Status Solidi B 251, 2104 (2014).

[8] O. Tegus, E. Brück, L. Zhang, Dagula, K.H.J. Buschow, F.R. de Boer. Physica B 319, 174 (2002).

[9] V.K. Pecharsky, K.A. Gshneidner, Jr. Phys. Rev. Lett. 78, 4494 (1997).

[10] V.K. Pecharsky, K.A. Gshneidner, Jr. J. Appl. Phys. 86, 565 (1999).

[11] Ö. Çakir, M. Acet. Appl. Phys. Lett. 100, 202404 (2012).

[12] H. Wada, Y. Tanabe. Appl. Phys. Lett. 79, 3302 (2001).

[13] N.T. Trung, V. Biharie, L. Zhang, L. Caron, K.H.J. Buschow, E. Brück. Appl. Phys. Lett. 96, 162507 (2010).

[14] D.T. Cam Thanh, E. Brück, N.T. Trung, J.C.P. Klaasse, K.H.J. Buschow, Z.Q. Ou, O. Tegus, L. Caron. J. Appl. Phys. 103, $07 \mathrm{~B} 318$ (2008).

[15] S. Fujieda, A. Fujita, K. Fukamichi. Appl. Phys. Lett. 81, 1276 (2002).

[16] P. Entel, M.E. Gruner, A. Dannenberg. Mater. Sci. Forum 635, 3 (2010).

[17] V.D. Buchelnikov V.V. Sokolovskiy. Phys. Met. Metallogrh. 112, 633 (2011).

[18] V.V. Sokolovskiy, M.A. Zagrebin, V.D. Buchelnikov. Mater. Sci. Foundations 81, 38 (2015).

[19] L. Pareti, M. Solzi, F. Albertini, A. Paoluzi. Eur. Phys. J. B 32, $303(2003)$

[20] F.X. Hu, B.G. Shen, J.R. Sun, G.H. Wu. Phys. Rev. B 64, 132412 (2001).

[21] V.V. Khovailo, K. Oikawa, T. Abe, T. Takagi. J. Appl. Phys. 93, 8483 (2003).

[22] И.Е. Дикштейн, Д.И. Ермаков, В.В. Коледов, Л.В. Коледов, Т. Такаги, А.А. Тулайкова, А.А. Черечукин, В.Г. Шавров. Письма в ЖЭТФ 72, 536 (2000).

[23] V.A. Chernenko, E. Cesari, V.V. Kokorin, I.N. Vitenko. Scripta Met. Mater. 33, 1239 (1995).

[24] G.A. Malygin. Phys. Usph. 44, 173 (2001). 
[25] C.P. Bean, D.S. Rodbell. Phys. Rev. 126, 104 (1962).

[26] R. Zach, M. Guillot, J. Tobola. J. Appl. Phys. 83, 7237 (1998).

[27] R. Abeyaratne, S.-J. Kim, J.K. Knowles. Int. J. Solids Struct. 31, 2229 (1994).

[28] S. Fabbrici, G. Porcari, F. Cugini, M. Solzi, J. Kamarad, Z. Arnold, R. Cabassi, F. Albertini. Entropy 16, 2204 (2014).

[29] V.V. Khovaylo, V.D. Buchelnikov, R. Kainuma, V.V. Koledov, M. Ohtsuka, V.G. Shavrov, T. Takagi, S.V. Taskaev, A.N. Vasiliev. Phys. Rev. B 72, 224408 (2005).

[30] A.A. Cherechukin, T. Takagi, M. Matsumoto, V.D. Buchel'nikov. Phys. Lett. A 326, 146 (2004).

[31] Q.-M. Hu, Ch.-M. Li, R. Yang, S.E. Kulkova, D.I. Bazhanov, B. Johansson, L. Vitos. Phys. Rev. B 79, 144112 (2009).

[32] V.A. Chernenko, V.A. L'vov, S.P. Zagorodnyuk, T. Takagi. Phys. Rev. B 67, 064407 (2003).

[33] V. Khovaylo, V. Koledov, V. Shavrov, A. Korolyov, K. Skokov, M. Ohtsuka, H. Miki, T. Takagi. Proceedings of Second IIFIIR. International Conference on Magnetic Refrigeration at Room Temperature. Portoroz, Slovenia (11-13 April, 2007). P. 217.

Редактор Т.Н. Василевская 